\title{
Collecting big data with small screens: Group tests of children's cognition with touchscreen tablets are reliable and valid
}

\author{
Giacomo Bignardi $^{1}$ (D) Edwin S. Dalmaijer ${ }^{1} \cdot$ Alexander Anwyl-Irvine $^{1} \cdot$ Duncan E. Astle $^{1}$
}

Accepted: 15 October 2020 / Published online: 2 December 2020

(C) The Author(s) 2020

\begin{abstract}
Collecting experimental cognitive data with young children usually requires undertaking one-on-one assessments, which can be both expensive and time-consuming. In addition, there is increasing acknowledgement of the importance of collecting larger samples for improving statistical power Button et al. (Nature Reviews Neuroscience 14(5), 365-376, 2013), and reproducing exploratory findings Open Science Collaboration (Science, 349(6251), aac4716-aac4716 2015). One way both of these goals can be achieved more easily, even with a small team of researchers, is to utilize group testing. In this paper, we evaluate the results from a novel tablet application developed for the Resilience in Education and Development (RED) Study. The RED-app includes 12 cognitive tasks designed for groups of children aged 7 to 13 to independently complete during a 1-h school lesson. The quality of the data collected was high despite the lack of one-on-one engagement with participants. Most outcomes from the tablet showed moderate or high reliability, estimated using internal consistency metrics. Tablet-measured cognitive abilities also explained more than $50 \%$ of variance in teacher-rated academic achievement. Overall, the results suggest that tablet-based, group cognitive assessments of children are an efficient, reliable, and valid method of collecting the large datasets that modern psychology requires. We have open-sourced the scripts and materials used to make the application, so that they can be adapted and used by others.
\end{abstract}

Keywords Cognition $\cdot$ Reliability $\cdot$ Validity $\cdot$ Childhood $\cdot$ Tablet $\cdot$ Group testing

Traditional one-to-one cognitive and behavioral assessments remain the gold standard in most aspects of psychological assessment. However, many standardized tools can be costly to purchase, and time-consuming to administer and score. This constrains the possible sample size of most studies, which is increasingly at odds with using innovative new methods (e.g., machine learning) that require larger sample sizes, and against recent moves to ensure that psychological science is robust and replicable (Button et al., 2013; Munafò et al., 2017). Additionally, relying solely on one-to-one testing imposes other limitations that may be more specific to the sample being studied. For example, research with young children frequently utilizes small convenience samples of families with the time and interest to attend a lengthy one-to-one assessment in a research lab, jeopardizing external validity (Keiding \& Louis, 2016).

Giacomo Bignardi

giacomo.bignardi@mrc-cbu.cam.ac.uk

1 MRC Cognition and Brain Sciences Unit, University of Cambridge, 15 Chaucer Rd Cambridge CB2 7EF UK
For research that requires detailed cognitive performance metrics with school-aged children, one alternative datacollection approach is to test groups of children in schools. This approach may reduce obstacles to research participation, which is especially important for recruiting groups that are typically under-represented in research, such as low socioeconomic status families (Jacobsen, Nohr, \& Frydenberg, 2010; Sakshaug, Schmucker, Kreuter, Couper, \& Singer, 2016; Winding, Andersen, Labriola, \& Nohr, 2014).

Despite the widespread adoption of group testing in education (e.g., SATs, GRE), it has been less widely adopted in developmental research. This might be explained by concerns about reliability, distractibility, and low motivation during group tests (Gregory, 2014; Murphy \& Davidshofer, 2004). Auditory distractions such as acoustic noise can impact task performance in children (Joseph, Hughes, Sörqvist, \& Marsh, 2018; Röer, Bell, Körner, \& Buchner, 2018). However, classroom noise may not impact test reliability nor decrease intertask correlations (Kanerva et al., 2019).

When adapted well, group tests can have excellent psychometric properties. For example, the widely used assessment tool WAIS-R has been adapted into a pencil-and-paper group 
test for ages $16+$, the Multidimensional Aptitude Battery (MAB-II). MAB-II test-retest reliabilities and task correlations to individually tested WAIS counterparts are high (Harrell, Honaker, Hetu, \& Oberwager, 1987; Jackson, 1998; Luciano et al., 2003). For young children, the wellvalidated CAT-4 pencil-and-paper group assessment covers quantitative, nonverbal, and spatial reasoning domains, also reports a high test-retest reliability (GL Assessment, 2012). However apart from a few exceptions (e.g., Brankaer, Ghesquière, \& De Smedt, 2017), many popular assessments are yet to be adapted for group testing.

Whilst pencil-and-paper assessments may be the simplest tests to create for groups, they are limited in the types of tasks that can be administered, and the types of outcomes that can be measured (e.g., no reaction times or dynamic measures), and require laborious manual scoring. Many of these limitations are addressed by using computerized tasks. There may be specific benefits for using computerized tasks, over and above their paper-and-pencil counterparts. For example, they permit audio instructions and reminders, reducing dependence on reading skills. Existing studies have not found large differences in performance in pencil-and-paper compared to computerized versions of common tasks (Piatt, Coret, Choi, Volden, \& Bisanz, 2016; Robinson \& Brewer, 2016). But computerized assessments can come with multiple technical challenges of their own. For example, relying on a school's IT system for administering tests can be hampered by bandwidth and browser issues (e.g.,Wassenaar et al., 2019).

Touch-screen tablet computers are another option for administering group cognitive tests and benefit from being relatively inexpensive and highly portable. The touch-screen interface of tablets is also easy to use: even children aged 2-4 can accurately complete basic tasks (Azah, Aziz, Syuhada, \& Sin, 2014; Semmelmann et al., 2016). Preliminary evidence suggests that young children prefer tablet assessments relative to pencil-and-paper tests (Piatt et al., 2016). Automated, computerized tasks are also less likely to suffer from experimenter bias and errors in scoring and administration (Chapman, Benedict, \& Schiöth, 2018; Styck \& Walsh, 2016). Selfguided tests generally require less training to administer, and can thus be more easily implemented in large cohort studies (Bhavnani et al., 2019). However, few tools currently exist specifically designed for testing children in groups, and little psychometric work exists validating these assessments.

Well-established tablet applications for child cognitive testing exist, however they are specifically designed for a researcher to provide instructions and closely monitor adherence. The NIH Toolbox is designed for testing cognition across the lifespan, initially tested in a large sample aged between 3 and 85 years (Weintraub et al., 2013), and has since been incorporated into other large research cohorts (Akshoomoff et al., 2014; Thompson et al., 2019). For children aged 2 to 5 , the Early Years Toolbox contains five cognitive tests with high internal consistency (Howard \& Melhuish, 2017).

Research on tablet applications specifically designed and validated for group-testing children's cognitive abilities, has been relatively mixed. Pitchford \& Outhwaite (2016) tested a sample of Malawi and UK school children, aged 4 to 12 on seven cognitive assessments including short-term memory, working memory, and mathematics ability. Reported task reliability varied between $5 \%$ (working memory) to $73 \%$ (mathematics). The varying reliabilities of tasks highlight the importance of task design and highlight the importance of validation of novel measures. In contrast, Kanerva et al. (2019) report on two novel tablet-based working memory tasks in a large sample of 12-year-olds, finding a moderate correlation between tests $(r=.44)$ and correlations to school grades $(r=.42 \& r=.36)$.

\section{Study overview}

The present study evaluated the effectiveness of using selfguided tablet-based cognitive assessments to collect largescale datasets, using group testing in school classrooms. While the code and materials used to generate the application are publicly shared and freely available for researchers to use, our primary goal here was to evaluate the general approach. The cognitive assessment was created as part of a large longitudinal study of children aged 7-9 years. The broader study aimed to collect a large, demographically representative dataset comprising behavioral, educational, cognitive, mental health, demographic, home environment, and teacher-rated measures. One group of children completed the assessments in groups in classrooms, and a smaller second group completed the same assessments individually in a laboratory setting, along with standardized cognitive tests (WASI-II).

We aimed to include a broad set of assessments in the application, which were divided into core and supplementary tasks. Core tests were chosen to tap key domains of cognition that have been highlighted as important for learning and school progress (Holmes, Bryant, \& Gathercole, 2019), and could also be easily adapted for tablet use. These included: reading and arithmetic fluency, short-term memory, matrix reasoning, visual search speed, and number discrimination. We note that this list is not exhaustive. The supplementary tests were more novel, and included at the end of the battery, so not all children may have completed these if testing time ran short. Evaluation of the tablet assessments is divided in three sections: reliability, predictive validity, and measurement invariance.

Reliability Our first goal is to determine the reliability of the cognitive tasks. No psychological construct can be measured perfectly without measurement error. Reliability coefficients 
specify the proportion of variance in an outcome that can be attributed to "true" differences between individuals, as a proportion of total variance (true and error variance; Revelle \& Condon, 2018). Reliability is a function of both task and sample, as samples with more restricted cognitive variability (e.g., because of a narrower age range), have lower variance in true scores, which thus makes up a smaller proportion variance in the observed score. Reliability was assessed via internal consistency metrics, which depend on correlations between different items (or split-halves) on the same test.

Predictive validity Unlike reliability, the concept of validity remains philosophically fraught, with no universally agreed definition (Gregory, 2014; Lissitz, 2009; Markus \& Borsboom, 2013). There is no single test for validity, but rather multiple sources of evidence should be acquired (AERA, APA, \& NCME, 2014). The first way we gathered validity evidence was to estimate how strongly the tablet assessments predict teacher-rated academic skills. As Wasserman (2018, pg. 11) notes, academic ability has "long been considered an independent criterion measure of intelligence", and indeed many of the first cognitive tests were developed for educational decision making. The strong correlation between cognitive tests and academic performance remains one of the most robust findings in the individual differences literature (Mayer, 2011; Roth et al., 2015). These effects likely go both ways, with education also improving cognitive abilities (Ritchie \& Tucker-Drob, 2018). Secondly, we estimated how strongly the tablet assessments predict a traditional standardized assessment tool (WASI-II; McCrimmon \& Smith, 2013). In this case, children completed both assessments individually at our laboratory, but the tablet assessments remain fully automated requiring no researcher administration.

Measurement invariance Does testing children in a group alter the psychological construct being tested? For example, if children are more inattentive when performing the tests in a classroom setting, this could lead to increased measurement error and alter inter-task correlations. Multi-group confirmatory factor analysis was used to compare measurement properties of the tests when the application is used in individual or group testing (Millsap \& Kim, 2018; Putnick \& Bornstein, 2016). This approach requires an initial measurement model to compare across groups. Various taxonomies of cognitive abilities permeate the literature, along with different labels (e.g., executive functioning, intelligence, etc.). No consensus prevails regarding the optimal factor decomposition of cognitive task data (e.g., Rey-Mermet, Gade, Souza, Bastian, \& Oberauer, 2019; Karr, Areshenkoff, Rast, Hofer, Iverson, \& Garcia-Barrera, 2018). Before assessing measurement invariance, we used exploratory methods to examine the factor/component structure of the tasks in the battery.

\section{Methods}

\section{Open software and data}

The study protocol was approved by the University of Cambridge Psychology Research Ethics Committee (PRE.2017.102). An Open Science Framework (OSF) repository (www.osf.io/xhejz/), contains analysis scripts, and the application materials and scripts. The original study data is not publicly available due to ethical constraints, but can be shared on request. A synthetic dataset generated using the synthpop R package (Nowok, Raab, \& Dibben, 2016) is openly available in the OSF repository.

The RED-App was programmed by the authors using the Unity Game Engine. One benefit of using a game engine like Unity is that the project can be exported to other platforms (e.g., Windows, Android, macOS), enhancing its general applicability. In addition, running the experiment in an online browser with a touch screen device can lead to decreased display and response recording accuracy, and relies on a consistent internet connection (Bridges, Pitiot, MacAskill, \& Peirce, 2020; Pronk, Wiers, Molenkamp, \& Murre, 2020). Materials have been shared for others to adapt or create new tasks within the current app framework.

\section{Participants}

The RED study was composed of two groups. A larger school cohort, which is the focus of this paper, were recruited from primary schools, and tested as a group in their usual classrooms, during 1-h sessions. Opt-out recruitment of children from eligible class year groups was conducted. We also recruited a second, smaller cohort tested at our laboratory in Cambridge, England. This cohort completed the same tablet cognitive assessments, along with brain scans (both structural and functional magnetic resonance imaging, and resting and task-based magnetoencephalography), a parent questionnaire, and traditional standardized cognitive assessments (WASI-II).

The RED school cohort is composed of 535 children who have completed at least one assessment, from six schools, and 22 classroom groups. Schools were located in the East of England. Testing occurred between June 2018 and March 2019. Participants from the school cohort were aged from 7.29 to 9.87 years $(\mathrm{M}=8.59$ years, $\mathrm{SD}=0.66)$. The $\mathrm{RED}$ laboratory cohort is composed of 92 children $(\mathrm{M}=8.49$ years, $\mathrm{SD}=0.84$ ) who completed the tablet assessments. There were no exclusion criteria for participation in the large school cohort, although data was omitted where schoolteachers believed a child could not independently complete the tasks without help from a teaching assistant. Both groups had a similar mean neighborhood deprivation $\left(r_{b}=.031,95 \% \mathrm{CI}\right.$ $[-.05, .11])$, measured using the England Index of Multiple Deprivation (Ministry of Housing, Communities, and Local 
Government, 2019). The distribution of deprivation in both cohorts is provided in the OSF repository, under supplementary figures.

Because data are drawn from a large longitudinal study, sample size was not determined from a power-analysis for the analyses presented here. We planned a sample size of 600-800 and 100 for the school and laboratory cohorts, respectively. Typical difficulties in recruiting schools applied and limited the cohort size through dropout of individual head teachers after their academy head agreed to take part, and unexpected scheduling conflicts that required schools to drop out after initially agreeing to take part. Practical constraints in testing enough participants before a cut-off date (September 2019 , to allow time for a 2-year follow-up investigation), limited the size of the laboratory cohort.

\section{Tablet cognitive assessments}

Thirty Apple iPads were used (on 9.7-inch, $1536 \times 2048$ resolution screens, model numbers: A1474, A1566, and A1822) for testing. After the first testing session, privacy filters were added to iPads to reduce distractions to children from nearby tablets. Children were also given large over-ear headphones to reduce external distractions and hear task instructions. We provide a brief description of each task below (see Fig. 1).

All tasks are presented in a fixed order, detailed in the accompanying GitHub repository (note the same order as presented here), so that more tasks at start of the battery were more likely to be completed. Completion rates for the two academic achievement tasks (Reading \&
Arithmetic fluency) and 5 "core" cognitive tasks (Visual Search Speed, Forward Digit Recall, Dot-Matrix Recall, Matrix Reasoning, \& NSND) were high, but lower for other tasks (see Table 1). In designing the task order, we also aimed to alternate less subjectively enjoyable tests (e.g., matrix reasoning) with more enjoyable tests (e.g., Visual Search Speed). Some tests incorporated feedback and gamification elements which we hypothesized would increase engagement with particularly repetitive tasks, whilst other tests modeled on already standardized tasks (e.g., the memory tasks) did not. Three "quiz" rounds including questionnaires were also interspersed between tasks.

Visual search speed Children are presented with an array of cartoon cat faces that are either smiling or neutral, over two rounds. In both rounds, they are asked to tap all the smiling cat faces as quickly as possible. A round ends if all smiling cats were found, the stop button is pressed, or two minutes have passed. In the first round, tapping a smiling cat produces auditory feedback ("meow!") over the headphones, and visibly marks it with a red cross. The second round is identical but tapping a smiling cat does not leave a visual mark, requiring children to remember targets they previously clicked. Cancellation tasks feature in various large cognitive batteries (e.g., Woodcock-Johnson-II) and are thought to assess processing speed and visual attention (Dalmaijer, Van der Stigchel, Nijboer, \& et al., 2015). The task was scored by calculating the $80 \%$ Winsorized (trimming the top and bottom $10 \%$ of values) mean time taken between successful cancellations in the "marked" cancellation round.

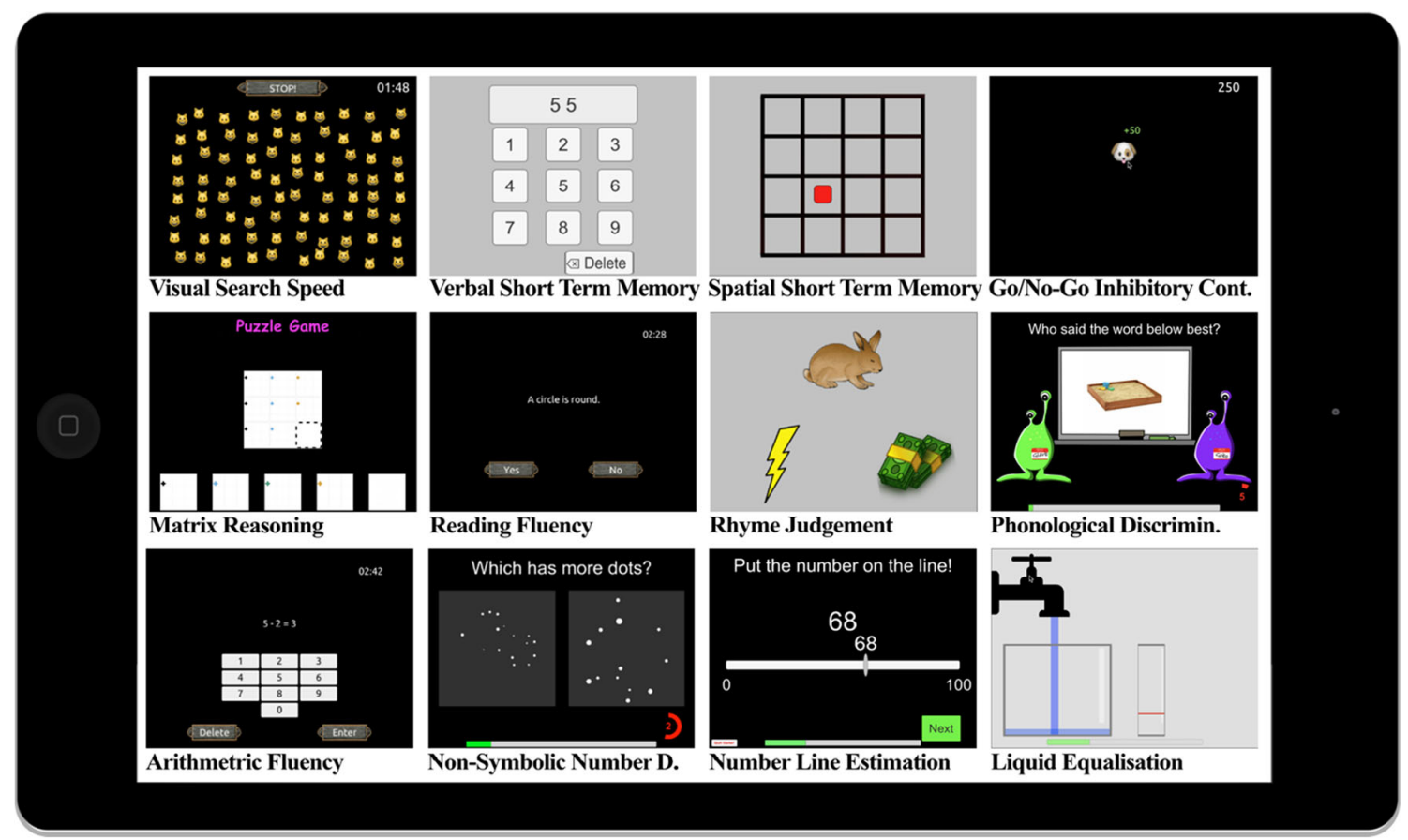

Fig. 1. Screenshots from the novel tablet assessments 
Table 1 Descriptive statistics for tablet cognitive assessments

\begin{tabular}{|c|c|c|c|c|c|c|c|c|c|c|c|}
\hline \multirow[t]{2}{*}{ Task name } & \multirow[t]{2}{*}{$N$} & \multicolumn{4}{|c|}{ Predictive validity } & \multicolumn{3}{|c|}{ Reliability } & \multicolumn{3}{|c|}{ Time taken } \\
\hline & & $r$ & LB & UB & $b^{*}$ & $\omega$ & LB & UB & $10 \%$ & $50 \%$ & $90 \%$ \\
\hline Visual Search Speed & 526 & -.38 & -.46 & -.30 & -.35 & .74 & .68 & .78 & 1.59 & 2.18 & 3.30 \\
\hline Verbal Short-Term Memory & 532 & .43 & .35 & .51 & .40 & & & & 2.28 & 4.36 & 6.82 \\
\hline Spatial Short-Term Memory & 532 & .45 & .37 & .52 & .41 & & & & 1.55 & 3.05 & 5.30 \\
\hline G/N-G - D' & 362 & .18 & .06 & .30 & .19 & .53 & .43 & .62 & 1.70 & 4.32 & 4.68 \\
\hline G/N-G - Omission Errors & 362 & -.41 & -.50 & -.30 & -.36 & .71 & .65 & .77 & 1.70 & 4.32 & 4.68 \\
\hline G/N-G - Commission Errors & 362 & -.13 & -.24 & -.01 & -.07 & .60 & .51 & .67 & 1.70 & 4.32 & 4.68 \\
\hline Matrix Reasoning & 515 & .48 & .40 & .55 & .46 & .76 & .73 & .78 & 2.55 & 4.17 & 6.38 \\
\hline Reading Fluency & 535 & .63 & .57 & .69 & .66 & .87 & .85 & .89 & 3.15 & 3.20 & 3.32 \\
\hline Rhyme Judgement & 488 & .27 & .18 & .36 & .26 & .68 & .59 & .76 & 1.11 & 3.52 & 4.25 \\
\hline Phonological Discrimination & 275 & .26 & .12 & .39 & .23 & .72 & .67 & .78 & 4.73 & 5.32 & 6.54 \\
\hline Arithmetic Fluency & 535 & .55 & .48 & .61 & .52 & .89 & .87 & .91 & 3.48 & 3.53 & 3.65 \\
\hline Non-Symbolic Num. Discrim. & 515 & .45 & .37 & .53 & .42 & .85 & .83 & .86 & 3.32 & 4.00 & 4.72 \\
\hline Line Estimation & 193 & .53 & .42 & .63 & .53 & .92 & .90 & .94 & 2.20 & 3.22 & 4.44 \\
\hline Liquid Equalization & 348 & .42 & .32 & .51 & .41 & .88 & .85 & .90 & 3.02 & 3.53 & 4.21 \\
\hline
\end{tabular}

Note: this table only includes data from the school cohort. Predictive validity was assessed by the linear Pearson correlation with teacher-rated academic ability, along with the lower and upper bounds of a 95\% confidence interval (LB, UB). We also report the standardized regression coefficient ( $\left.b^{*}\right)$ for each task predicting academic ability whilst accounting for age and normalized neighborhood deprivation, in a multivariable regression performed separately for each task. The $10 \%, 50 \%$ (median) and $90 \%$ percentiles of time taken in minutes to complete each task are reported in the final three columns.

Verbal short-term memory We implemented a standard digit recall memory test (e.g., Alloway, 2007). Children are presented with a series of digits (visible on-screen and spoken aloud over the headphones) in a sequence, and are asked to repeat the sequence on a virtual number pad. Sequence length begins at three digits and after getting at least four correct (out of a possible six trials) they move up a level in span length (up to a maximum of nine digits). The task was scored using the number of correct sequences performed.

Spatial short-term memory We implemented a standard dotmatrix memory test (e.g., Alloway, 2007). Children are presented with an empty $4 \times 4$ matrix, and a series of red square dots light up in each box in a sequence. Children are then asked to repeat the sequence by pressing the relevant squares in each part of the matrix in the same order. The sequence length then increases using the same rules like the forward digit recall task, and is scored identically.

Go/no-go inhibitory control Children are asked to quickly tap a target stimulus (a dog emoji) when it appears on the screen, and to avoid pressing the distractor (a poop emoji). The order and timings of stimulus presentation were randomized. Pressing the target stimulus elicited a barking noise, and pressing the distractor elicited a fart noise. A feedback numerical "score" was presented on the top right corner which increased 50 points for pressing a target stimulus and reduced 150 points when pressing the distractor. We scored three outcomes: the number of omission errors (failure to tap target), the number of commission errors (tapping a distractor stimulus), and the sensitivity index (d').

Matrix reasoning A two-part matrix reasoning assessment was used to measure abstract problem-solving skills. In the first part, children are presented with a series of abstract figures and are asked to select which of five drawings would appear next in the sequence. In the second part, children are presented with five abstract figures and asked to identify the figure that is different from the others. The task was scored by estimating latent ability from an item response theory analysis of each trial (detailed in the Data Analysis section).

Reading fluency Children are asked to read short statements (mean character length $=30.8$ ) and decide if the sentence is correct or incorrect as quickly as possible. The statements are designed to assess reading skill rather than knowledge, so are relatively straightforward (e.g., "A dog can fly"). The task ends after $3 \mathrm{~min}$ and is scored by the number of correct responses minus the number of incorrect responses.

Rhyme judgement Phonological awareness was assessed with a rhyme judgement task. Each trial begins with a recorded voice stating, "does X rhyme with Y, or Z", with visual depictions of each word being presented in line with the audio. The child responds by clicking on the best rhyming image. The presentation of the correct rhyme pair is left/right 
balanced across the whole set. The rhyming targets consisted of two or three syllables, and could include 'near-rhyme' distractors for added difficulty. All words were matched for word-frequency, age-of-acquisition and concreteness (so they could be easily identified by images) using the Kuperman, Stadthagen-Gonzalez, \& Brysbaert (2013) database. Initially, the task consisted of 22 items, though following an initial analysis of the task data we reduced the number of items to six (see Results). The task was scored identically to the matrix reasoning task.

Phonological discrimination A phonological discrimination task adapted from Davis et al., (2019) was used to test children's perceptual acuity of speech. For each trial, the child hears a female-sounding "teacher" pronounce a monosyllabic noun (e.g., "fan"), which is also visually presented on a "whiteboard". They then hear two malesounding aliens on either side of the whiteboard consecutively repeat either the same or a different word with a single articulatory feature change ("van"). The child is asked to tap on the alien which repeated the teacher's word most accurately. Task difficulty is modulated by altering how similar the Alien's words sound. We selected 44 concrete word pairs and used a range of difficulties, using data and materials provided by its authors. Response accuracy was indicated by a bell with a high (correct) or low (incorrect) pitched tone after selection. The task was scored identically to the matrix reasoning task.

Arithmetic fluency Children are asked to solve as many arithmetic problems as possible in $3 \mathrm{~min}$, using a virtual number pad. The task ends after $3 \mathrm{~min}$ and is scored identically to the reading fluency test.

Non-symbolic number discrimination (NSND) This task requires children to pick which of two clouds of dots is more numerous, assessing a visual number sense (Odic \& Starr, 2018). Task difficulty is modulated by altering the ratio of numbers presented, as larger differences are easier to discriminate. After three simple training rounds including verbal feedback, 116 testing trials are presented and used for scoring. Six different ratios of dots are used $(4,2,1.5,1.3,1.2,1.1)$. Response accuracy was indicated by a bell with a high (correct response) or low (incorrect response) pitched tone after selection. Gebuis \& Reynvoet (2011) script was used to generate stimuli so that the number of dots presented is minimally correlated with dot size or spatial extent. The task was scored identically to the matrix reasoning task.

Line estimation Children are asked to mark points on a number-line, anchored at 0 and 100 , where a given number would be located. Forty trials were presented in a fixed order. The task is scored by ranking each child on each problem by accuracy (absolute difference between target number and the line position selected), and normalizing the percentile ranks on each trial using the procedure described below. Then, the average normalized $z$-score on the 40 trials is calculated for each child and normalized again.

Liquid equalization This task is an adaptation of Piaget's equalization test (Silverman \& Rose, 1982). Children are presented with two 2D empty glasses of water. They are asked to pour an amount of liquid into a left glass, that would fill the glass on the right up to a red line marking. Both the width of the glass on the right and the height of the red line change on each trial, so children have to utilize knowledge of 2D areas. On each trial a feedback "score" is given depending on the pouring accuracy. The task is scored identically to the line estimation task.

\section{Other assessments}

Teachers completed a shortened Academic Performance Questionnaire (APQ; Bennett, Power, Eiraldi, Leff, \& Blum, 2009). We selected the three items, "compared to the average student in your class, how well is the child: (1) able to read orally, (2) able to write short stories and class assignments, (3) able to perform math calculations". The second item was added after visiting the first school, so is missing for 30 children. Teachers answered questions using a digital visual analogue scale, anchored at "well below average" to "well above average". Sixteen teachers fully completed the questionnaire, with APQ information on 446 children in total. Mean imputation on the APQ was used for the 30 children with one missing item. Teacher's responses across the three items were highly consistent $(\omega=.97,95 \%$ CI $[.94, .96])$. Children in the laboratory cohort completed the WASI-II vocabulary and reading subtests. For both variables, a factor score was estimated using the ten Berge method in the psych $\mathrm{R}$ package (Grice, 2001).

\section{Data analysis}

All analyses were conducted using R (v3.6.2), with scripts and associated files openly available (https://osf.io/xhejz/). Two approaches were taken to ensure computational reproducibility. The R package renv (Ushey, 2020) was used to manage $\mathrm{R}$ package dependencies, and will install the required the packages and versions used here. We also provide a docker container which captures a compatible computational environment for running the scripts (Nüst, Eddelbuettel, Bennett, Cannoodt, Clark, Daróczi, ... Petegem, 2020).

All task outcomes (apart from the Go/No-Go d') were normalized. First, percentile ranks were estimated (ranging between 0 \& 1) for scores on each test. A child who scored higher than $90 \%$ of their peers would have a percentile rank of 0.9. The percentile ranks were than mapped onto a standard 
normal distribution using the normal quantile function. This typical procedure for standardizing score limits the influence of univariate outliers, and maps raw scores onto an easily interpretable scale (Gregory, 2014).

Reliability For tasks where all children complete an identical set of items, Revelle's omega total $(\omega)$ was used to determine internal consistency from item-level factor analyses (McNeish, 2018). The R package psych (v1.8.12, Revelle, 2018) was used to estimate omega (setting number of group factors to 1), and custom code implemented non-parametric percentile bootstrapping to estimate confidence intervals with 1000 resamples. For timed tests with number (in)correct outcomes (Go/No-Go, Reading Fluency, Arithmetic Fluency), the split-half reliability was estimated also using omega. Internal consistency could not be estimated this way for the short-term memory tasks because the task ended early when participants gave consecutive incorrect responses.

For tasks with binary correct/incorrect trial outcomes (Matrix Reasoning, Rhyme Judgement, Phonological Discrimination, Non-Symbolic Number Discrimination), we also estimated reliability using item response theory (IRT). One advantage of IRT analyses is that measurement error is estimated conditional on true ability level, rather than a constant. For example, a multiple-choice mathematics test with only very simple questions may be good at discriminating between individuals with very poor mathematics ability, but ineffective at discriminating between above-average students. The mirt R package (v1.30, Chalmers, 2012) was used to fit a two-parameter (slope and difficulty) IRT model, using the Oake's method for estimating parameters. The guessing parameter was fixed at the reciprocal of the number of choices on a given test (e.g., 25\% for a four-choice test). Items with a negative discrimination parameter were removed from final models. Expected a-posteriori factor score estimation was used to calculate children's performance on each task, using the mirt::fscores function.

Predictive validity Multivariable regression was used to estimate the extent to which numerous tablet cognitive assessments can jointly explain teacher-rated academic ability, and the WASI-II scores. The main outcome is the percentage of variance that can be explained by the tablet assessments. The adjusted $R^{2}$ metric was used which corrects for bias in the standard $R^{2}$ (Ohtani, 2000). A bias-corrected and accelerated bootstrap 95\% confidence interval for $R^{2}$ was estimated using the "boot" R package (6000 resamples; Davison \& Hinkley, 2019).

For predicting teacher-rated academic ability, we separately reported prediction accuracy when using the two "achievement" tests (reading and arithmetic fluency) or the other five core cognitive tests (Search Speed, Matrix Reasoning, DotMatrix Working Memory, Forward Digit Span \& NSND). These analyses were separated as we were interested in the extent to which the Academic Achievement outcome is best explained by academic achievement tablet measures, compared to the more general cognitive measures. All seven measures were also combined to estimate the overall variance explained by tablet tasks. Due to low completion rates, we did not include supplementary tests in these analyses because the high rate of non-completion would reduce the sample size for the analyses. Missing data were imputed for participants with only one missing tablet assessment out of the seven. The classification and regression trees imputation method from the MICE R package was used (Van Buuren \& GroothuisOudshoorn, 2011).

The number of children each teacher rated varied from 10 to 32 , with a mean of 26.24. If teachers systematically over- or under- estimated children's performance, then observations cannot be treated as statistically independent. To account for potential non-independence, the R package lme 4 was used to fit random-effect models with restricted maximum likelihood estimation (Bates, Mächler, Bolker, \& Walker, 2015). The intraclass correlation coefficient (ICC), the proportion of variance explained by the random effects, is reported for each model. Confidence intervals were calculated using lme4's percentile bootstrap method. We used the same imputed data as described above.

The same approach was taken to estimate prediction accuracy for WASI-II. Note that because children were tested in individual sessions, more tasks were completed overall and there was fewer missing data on some tasks. Therefore, in the regression model all task outcomes in Table 1 were used apart from Line Estimation, Rhyme Judgement and Liquid Equalization which has the lowest completion rates $(<58 \%)$. For the remaining 11 task outcomes, the same imputation method was used, but children were excluded if tablet cognitive data was missing for more than three variables.

Measurement invariance We assessed measurement invariance using standard psychometric procedures employed in the R functions Lavaan::cfa (v0.6-5, Rosseel, 2012) and semTools::measurementInvariance (v0.5-2, Jorgensen, Pornprasertmanit, Schoemann, \& Rosseel, 2018), presented in Table 2. This implements a standard multi-group confirmatory factor analysis approach for checking measurement invariance across the school and laboratory-tested cohorts. It works by running confirmatory factor analyses in both groups, and over a series of model changes, it constrains additional parameters (i.e., loadings, intercepts, residual and means) to be equivalent across groups. Constrained models are compared against the unconstrained "configural model", to compare model fit indices. Multiple model fit indices are presented, including Chi-square $\left(\chi^{2}\right)$ and its corresponding $p$ value, comparative fit index (CFI), root mean square error of approximation (RMSEA), and Akaike Information Criterion (AIC). 
Table 2 Assessment of measurement invariance across the group-tested $(N=92$, school) and individually tested $(N=535$, laboratory) cohorts

\begin{tabular}{|c|c|c|c|c|c|c|c|c|c|c|c|c|}
\hline \multirow[t]{2}{*}{ Models } & \multicolumn{3}{|l|}{$x^{2}$} & \multicolumn{4}{|c|}{ Comparative Fit Index (CFI) } & \multicolumn{4}{|c|}{ Root mean square error of approximation } & \multirow[t]{2}{*}{ AIC } \\
\hline & $\chi^{2}$ & $d f$ & $p$ & CFI & $\Delta$ & LB & UB & RMSEA & $\Delta$ & LB & UB & \\
\hline 1. Configural & 63.4 & 28 & & .971 & & & & .0635 & & & & 11012 \\
\hline 2. Loadings & 73.8 & 34 & .107 & .967 & -0.004 & -.024 & .001 & .0611 & -0.002 & -.010 & .010 & 11010 \\
\hline 3. Intercepts & 98.3 & 40 & $<.001$ & .952 & -0.015 & -.038 & -.005 & .0682 & 0.007 & -.004 & .018 & 11023 \\
\hline 4. Residuals & 107 & 47 & .273 & .951 & -0.001 & -.023 & .001 & .0638 & -0.004 & -.008 & .005 & 11017 \\
\hline 5. Means & 113 & 48 & .015 & .947 & -0.004 & -.014 & .001 & .0657 & 0.002 & -.001 & .006 & 11021 \\
\hline
\end{tabular}

Note: The lower (LB) and upper bounds (UB) of the $95 \%$ confidence interval for changes $(\Delta)$ in CFI \& RSMEA are reported, calculated using nonparametric bootstrap resampling (3000 repeats). Random sampling was performed within each group separately for each iteration.

Conventionally, changes in CFI of less than -0.01 , or changes in RMSEA of greater than 0.01 , are interpreted as evidence of measurement invariance (Putnick \& Bornstein, 2016). The model with the lowest AIC is said to be best. To estimate uncertainty in CFI and RSMEA from sampling error, we estimated percentile bootstrap confidence intervals (3000 repetitions).

Because this requires a measurement model to compare across groups, the task factor structure was explored in the school cohort first. Parallel analysis and Velicher's minimum average partial correlation (MAP) method were used to determine the optimal number of components/factors to explain variance in task scores, using the psych R package. MAP finds the number of components that minimize the average squared residual correlations. Parallel analysis compares the variance explained by each factor/component, to the variance explained when the data has been randomly permutated (repeated 1000 times). Factors/components are kept until they explain equal to or less variance than in the permutated datasets.

\section{Results}

The time taken to complete each task is reported in Table 1 . For most tasks, $90 \%$ of children completed them in under 5 min, though some tasks such as matrix reasoning and verbal/ visual short-term memory took slightly longer.

\section{Reliability - omega total $(\omega)$}

Descriptive information on each task including internal consistency, are presented in Table 1. Most tasks have good reliability (between 68\% and 92\%), except for two of the Go/NoGo outcomes.

\section{Reliability - item response theory (IRT)}

The two-parameter IRT model failed to converge (within < 30,000 iterations) for the NSND task. The larger number of trials (116) in NSND requires estimating many parameters (232), perhaps requiring a greater sample size. Instead for NSND, a simpler Rasch model was used estimating only a difficulty parameter.

A Test Reliability Function (Fig. 2) for each task was computed using the mirt package, where the $x$-axis presents latent ability on a uniform scale. Despite adequate omega reliability, the IRT analysis demonstrates that the rhyme judgement task is largely poor at discriminating between children above the $20 \%$ ability percentile. Indeed, a strong ceiling effect is evident for this task, as median participant accuracy was $95.4 \%$. Other studies report that ability to judge rhymes is at ceiling by the age of 5 years (Stanovich, Cunningham, \& Cramer, 1984,

Sumner, 2018), which we did not overcome by employing a greater number of syllables and near-rhyme distractors. This illustrates the value of IRT modeling, as the high reliability and validity metrics in Table 1 mask these limitations of the test.

Our subjective experience with the assessment overlapped with the above analysis of the rhyme judgement task during data collection. Consequently, we added the phonological discrimination test mid-way through data collection, to provide an additional, more sensitive test of phonological skills. We then reduced the number of trials to six in the rhyme judgement task. As a result, both tests were not fully completed by the whole sample. The other three assessments demonstrated adequate reliability across ability levels, though the Matrix Reasoning task would benefit from additional easier items for this age-group.

\section{Predictive validity: Academic ability}

Pairwise correlations with academic ability for all tests are reported in Table 1 and Fig. 3. We estimated how much variance in teacher-rated academic ability, measured with the APQ, could be explained by combining tablet assessments using multivariable regression. 


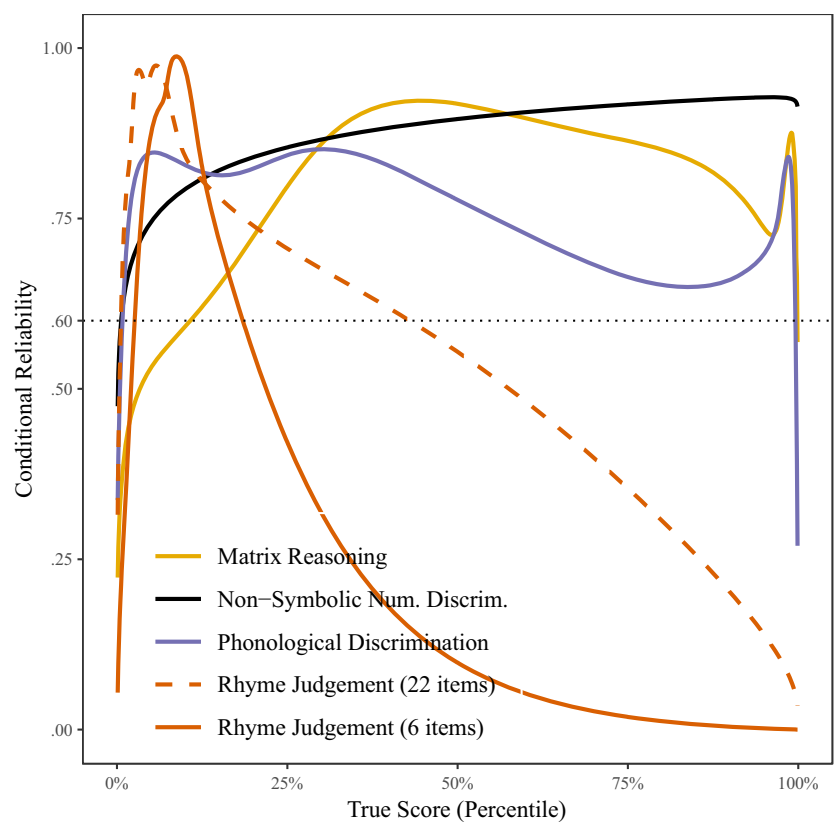

Fig. 2. Test reliability function, modeling measurement error as a function of latent ability, rather than a constant

The reading and arithmetic fluency tests alone predicted $44.9 \%$ of variance $\left({ }_{\mathrm{adj}} R^{2}=44.9,95 \%\right.$ CI $[.38, .51], d f=$ 411). The five main cognitive outcomes (Search Speed, Matrix Reasoning, Dot-Matrix Working Memory, Forward Digit Span \& NSND) predicted $39.5 \%$ of variance $\left({ }_{\text {adj }} R^{2}=\right.$ $39.5,95 \%$ CI $[.33, .47], d f=404)$. Combining all seven tablet assessments can explain just over half the variance in teacherrated academic ability ( ${ }_{\text {adj }} R^{2}=51.0,95 \%$ CI $[.44, .57], d f=$ 402).

Because teachers provided APQ ratings for multiple children, this may violate the independence of errors assumption. Therefore, we fitted a random intercept model with the seven tasks described above as fixed effects. The random intercept accounts for mean differences in teacher ratings between classrooms, and explains $6 \%$ of variance in outcomes $\left(\sigma^{2}=\right.$ $.066,95 \% \mathrm{CI}[.02, .14], \mathrm{ICC}=.060)$. Note that in the context of mixed linear modeling the ICC is not indicative of reliability. Importantly, the tablet assessments still explained just over half the variance in teacher-rated academic ability $\left(R^{2}=.55\right)$. Both fixed and random effects could explain $61 \%$ of variance in teacher ratings. Potentially there is a small tendency for teachers to over- or under-estimate ability, but the cognitive tests remain strong predictors of ability when accounting for this.

\section{Predictive validity: WASI-II}

Children in the smaller laboratory cohort completed both the tablet tasks and the WASI-II, a standardized cognitive test. We used multivariable regression again to predict the WASI-II factor scores. Due to the higher rates of task completion in the laboratory cohort compared to the school cohort, the three Go/No-Go outcomes and phonological discrimination tasks were also added to the linear models. The 11 task outcomes combined predicted $42.4 \%$ of variance in WASI-II factor scores $\left({ }_{\text {adj }} R^{2}=42.4,95 \%\right.$ CI $[.31, .59], d f=$ $62)$. This effect is large given the low correlation between WASI-II tasks, which resultingly have a low estimated reliability (omega total $=.49$ ).

\section{Measurement invariance: Task factor structure}

Before running measurement invariance analyses, it is important to establish the latent variable structure of the tasks. We used principal component analysis and exploratory factor analysis, using data from the school cohort only. Data from the seven tasks with the highest completion rates across the two samples were used, including: Visual Search Speed, Matrix Reasoning, Dot-Matrix Working Memory, Forward Digit Span, NSND and Arithmetic and Reading Fluency).

The parallel analysis (see Fig. 4) suggested that a single factor or component best explained task score variance, as extracting additional components did not explain more variance than would be expected in randomly permutated datasets. A single factor solution also minimized the MAP (0.034).

\section{Measurement invariance}

As the previous section suggested a single-factor solution, we fitted a multigroup confirmatory factor analysis (CFA) model with a single latent cognitive factor on same seven tasks, in school and laboratory RED cohorts. Measurement invariance analyses test whether model fit is worsened by constricting loading and intercepts to be equivalent across the two groups. Results are also presented in Table 2.

First, we fitted a configural model allowing all parameters to be freely estimated in each group. In Model 2 , task loadings on the latent factor are constrained to be equivalent across the two groups. This did not significantly reduce model fit $(p=$ $.107)$, and only marginally reduced comparative model fit $(\triangle \mathrm{CFI}=-.004)$ and root mean square error of approximation $(\triangle \mathrm{RMSEA}=-.002,95 \% \mathrm{CI}[-.008, .005])$ measurement fit indices. The AIC also suggests that Model 2 is the preferred model. This suggests that tasks measure latent ability equally well across the two groups. The third model constrains the intercepts of task performance on latent ability; the expected task scores when latent ability is zero. This tests whether group differences in task performance cannot be explained by latent ability alone. A significant $(p<.001)$ decrease in model fit is observed, with a moderate reduction in CFI $(\Delta \mathrm{CFI}=-.015,95 \%$ CI $[-.038,-.005])$, although the RSMEA did not increase above the conventional 0.1 threshold $(\triangle \mathrm{RMSEA}=.007,95 \% \mathrm{CI}[-.004, .018])$. The fourth model 


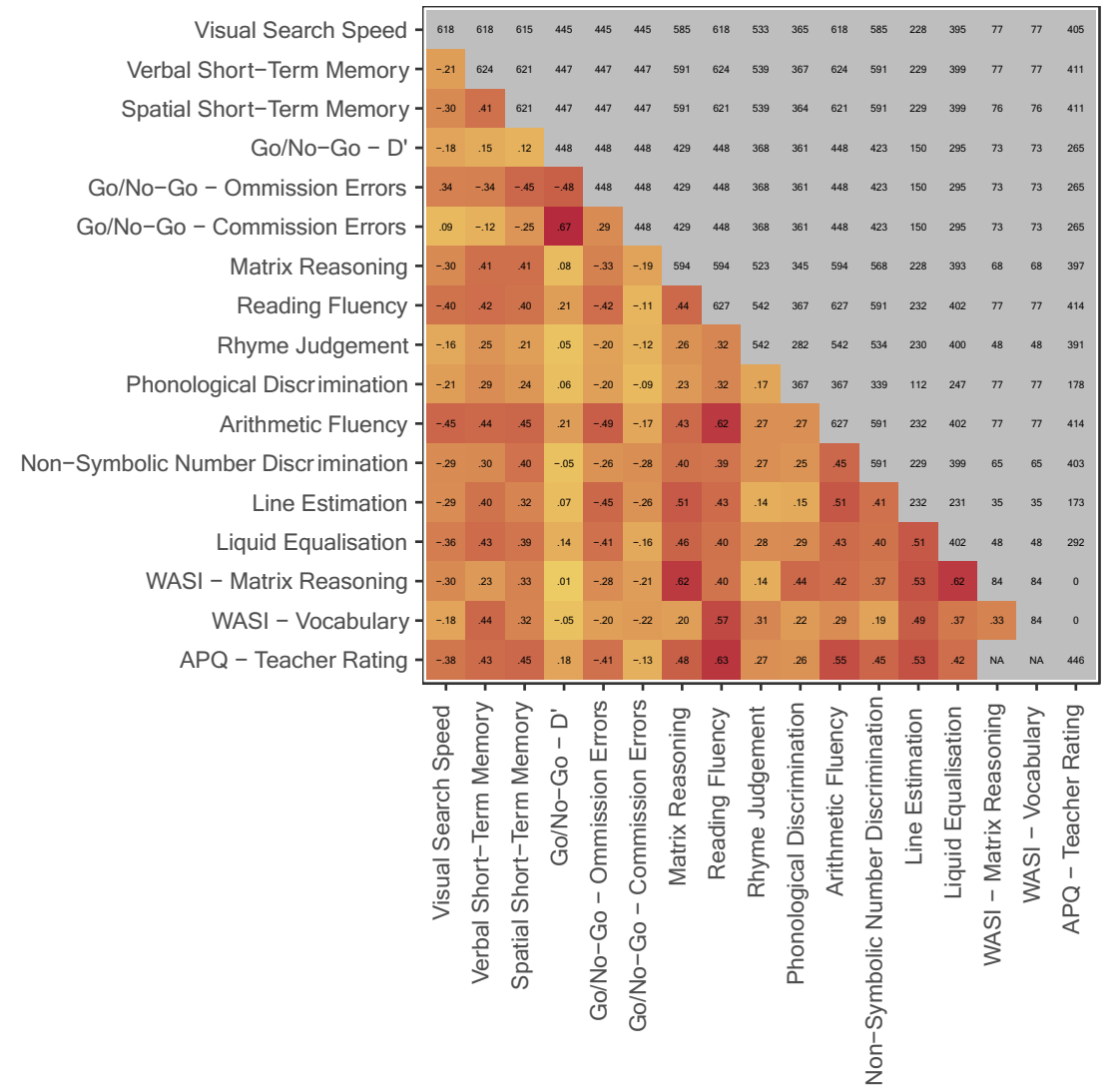

Fig. 3. Pearson correlation matrix of primary outcomes, including all RED participants from both school and laboratory cohorts

constrained the item residuals, the remaining variance in each item after partialing out latent ability. This did not change the $\mathrm{CFI}(\triangle \mathrm{CFI}=-.001,95 \% \mathrm{CI}[-.023, .001])$, or RMSEA more than the threshold amounts $(\triangle \mathrm{RMSEA}=-.004,95 \% \mathrm{CI}[-$ $.008, .005])$. The final model constrained the latent ability means to be equivalent. Again, this did not change the CFI $(\triangle \mathrm{CFI}=-.004,95 \% \mathrm{CI}[-.014, .001])$, or RMSEA more than the threshold amounts $(\triangle \mathrm{RMSEA}=.002,95 \% \mathrm{CI}[-.001$, $.006])$. The Chi-square test does suggest that a significant reduction in model fit occurred $\left(\chi^{2}=113, p=.015\right)$.

While there is evidence for metric invariance, that overall the tasks measure the latent g-factor equally well across the groups, there may other important measurement differences. Average performance (latent ability) and performance on

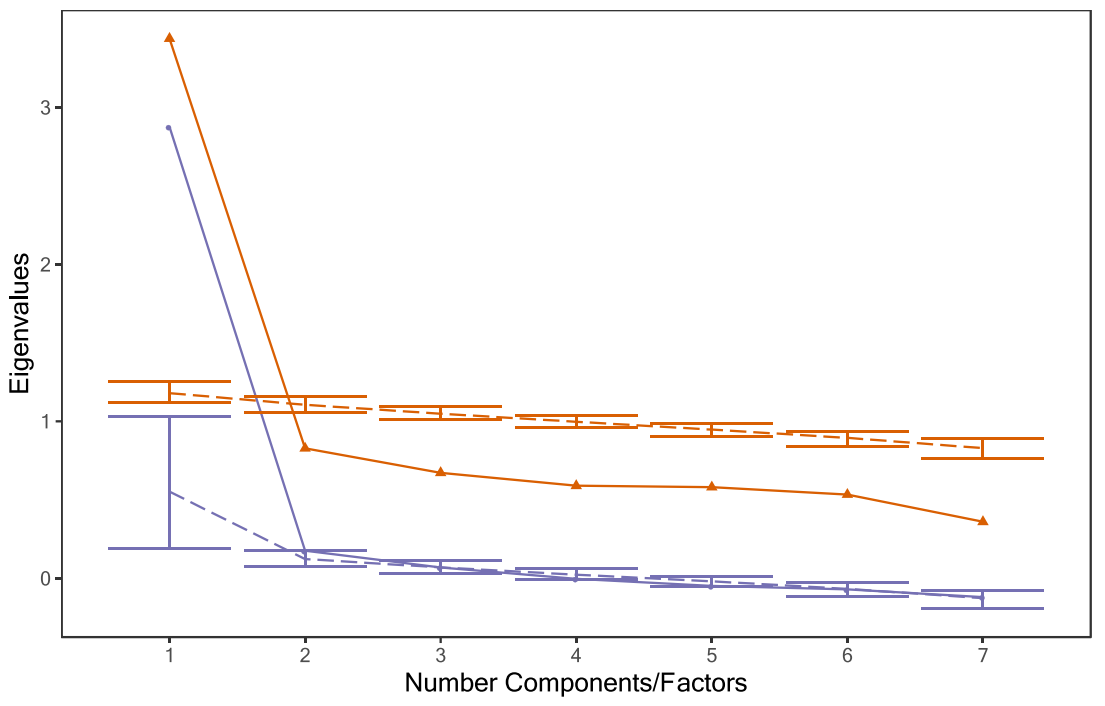

Fig. 4. Parallel analysis for all tasks, using data from school cohort only. Note. The bottom purple line represents successive factor analysis eigenvalues, and the top orange line for principal components. The dashed lines represent eigenvalues taken from resampled datasets. 
individual tasks (task intercepts) appear to be invariant. Inspection of Model 2 (Table 2) intercepts suggests that the lab group performed on average better on matrix reasoning (lab group intercept $=.381$, Bonferroni corrected $p=.005$ ). There was also a non-significant trend for higher performance in digit span (lab group intercept $=.257$, Bonferroni corrected $p=.053)$. Potentially, these tasks were more negatively impacted by distractions in the classroom.

Ultimately, these results do not show that raw scores on lab and individual testing can be directly compared. The relatively wide confidence intervals suggest more evidence is needed to draw robust conclusions. Crucially, however, there is also no evidence that scores in either cohort are more informative than the other, with no evidence of different task loadings across groups. Indeed, estimated internal consistency from the seven tasks in the laboratory (omega total $=.78,95 \% \mathrm{CI}[.70, .84]$ ) and school cohorts (omega total $=.82,95 \% \mathrm{CI}[.80, .84]$ ), are comparable.

\section{Discussion}

We found that 7 to 9 -year-olds can quickly and reliably complete cognitive assessments using an automated tablet application - requiring minimal researcher assistance. Ninety percent of the sample can complete the seven core tasks in under 34 min (Table 1). Reliability was high for most assessments, around 70-90\%. These assessments had strong predictive validity, explaining over half the variance in teacher's ratings of academic ability. This compares favorably to a meta-analytic estimate that standard intelligence tests predict just over $19 \%$ of variance in school grades (Roth et al., 2015). Some individual tasks had low reliability and predictive validity, and could be refined or replaced in future applications. For example, the item response theory analysis found that the rhyme judgement task was too easy for most children. Two outcomes from the Go/No-Go task had low reliability (53\% and 60\%), though this is not uncommon for inhibitory control tasks (Enkavi et al., 2019; Hedge, Powell, \& Sumner, 2018; Rouder, Kumar, \& Haaf, 2019). In a smaller laboratory group who completed the tablet assessments individually, tablet performance explained $43 \%$ variance in a traditional cognitive ability assessment (WASI-II).

Analyses of measurement invariance do not show that the tablet assessments are less reliable or predictive of latent ability when used as group tests in schools, compared to when assessments are completed individually. However, there were some small differences. Differing task intercepts indicated that the lab sample performed better on the matrix reasoning task, and that the two samples had different mean latent ability levels. Potentially, the contrasting sampling procedures between the two samples could explain differences, though it is uncertain why this would impact performance on an individual task. Potentially, the more distracting school environment may decrease performance on specific tasks that require more attention, such as matrix reasoning.

We expect our results can be replicated within certain boundary conditions. It is likely that using different tasks, or similar tasks with different stimuli or procedures will affect the individual reliabilities, inter-task correlations \& mean performance. For example, changes in stimuli sets used in non-symbolic number discrimination tasks can have strong impacts on measured performance (DeWind \& Brannon, 2016; Smets, Sasanguie, Szücs, \& Reynvoet, 2015). However, regardless of the specific tasks employed, single factors extracted from broad task batteries ("g-factors") tend to converge on very similar estimates of cognitive ability. Indeed, Spearman termed this phenomenon "the indifference of the indicator" (Johnson, Bouchard, Krueger, McGue, \& Gottesman, 2004; Thorndike, 1987; Vernon, 1989). Additionally, g-factors have been identified across a wide range of populations (Warne \& Burningham, 2019). Therefore, we expect general cognitive ability estimates derived from large, heterogenous batteries of tasks to be similar across studies, even when using somewhat different tasks.

Despite the success of our measurement tool, group testing presents additional, somewhat unique, challenges. Making sure that tasks are intuitive and easy to use without help is essential.

Although some of the tasks presented here included accuracy feedback and points systems, the current evidence is highly mixed regarding its effects on engagement (Attali \& Arieli-Attali, 2015; Ling, Attali, Finn, \& Stone, 2017) and accuracy (Beckmann, Beckmann, \& Elliott, 2009; Betz, 1977; Delgado \& Prieto, 2003). More broadly, the gamification of cognitive tests has been suggested to improve engagement and data quality, but the existing evidence base is limited (Dockterman et al., 2020; Lumsden, Edwards, Lawrence, Coyle, \& Munafò, 2016). We recommend extensive piloting under observation during the development of any self-administered tasks. The results from these assessments may not generalize if the testing environment is highly chaotic. Distractions can be minimized by using over-ear headphones and privacy screen filters on the tablet screen. Adding secondary tests or games at the end of a battery can keep children who finished early occupied, and avoid distracting others.

By including questionnaires in the application, children's environments and mental health can also be measured, allowing researchers to quickly generate evidence that is of current policy interest, for example on the effects of environmental exposures (e.g., Bignardi et al., 2020; Dalmaijer, Bignardi, Anwyl-Irvine, Smith, Siugzdaite, Uh, ... Astle 2019). One relatively unexplored application of group-based testing with tablets is as a costeffective "screener" for the early detection of cognitive difficulties. This could help recruitment of children with particular cognitive profiles for subsequent studies, or to identify children who 
might find learning more difficult in a conventional classroom setting, which would permit subsequent in-depth assessment and earlier targeted interventions (Gaab, 2019). However, screeners require higher evidence of reliability and practical utility. For example, even an "excellent" reliability of $90 \%$ would correspond to a $95 \%$ confidence interval of \pm 9.3 points (assuming an IQ-like scale with a standard deviation of 15; Revelle \& Condon, 2018). Further improvements to reliability could be possible by utilizing more sophisticated modeling techniques (Farrell \& Lewandowsky, 2018; Haines et al., 2020), or adaptive testing procedures (Harrison, Collins, \& Müllensiefen, 2017).

An alternative, promising approach to the one outlined here is to utilize remote online testing, and developmental researchers are increasingly exploring this option due to the COVID-19 epidemic disrupting laboratory research (Dillon, 2020). As popular online participant recruitment services do not include under 18-year-olds, researchers rely on slower, traditional recruitment approaches. As with other convenience samples, representativeness is an issue (McCredie \& Morey, 2019). One innovative approach is to utilize online digital educational content, for example Math Garden is a popular educational tool containing multiple games for children to practice math problems (Brinkhuis, Cordes, \& Hofman, 2020). Data gathered using these applications can produce large and detailed longitudinal datasets, which can be used to test learning theories (e.g., Hofman et al., 2018).

\section{Conclusions}

The importance of measurement is often overlooked in psychological research, which can be seen in the proliferation of "Questionable Measurement Practices" (Flake \& Fried, 2019). More efficient measurement practices, such as described in this paper, can also advance the field by allowing larger samples of cognitive data to be rapidly acquired, which are required for making robust inferences. Here, we outline a methodology for rapidly acquiring large datasets of cognitive data in school-aged children. With 2-4 researchers overseeing data collection, data on up to 30 children can be acquired in an hour, or 150 children in a school day. Cognitive data collected in this way has good reliability and validity evidence.

Author Note The Resilience in Education and Development (RED) Study is supported by grant TWCF0159 from the Templeton World Charity Foundation, and by the UK Medical Research Council. An earlier version of this article has been shared on OSF Preprints (https://osf.io/aw6c5/), and portions of the findings have been presented as posters at the 2019 British Neuroscience Association (BNA) and 2019 British Association for Cognitive Neuroscience (BACN) conference. We have no conflicts of interest to disclose.
Acknowledgements We would like to thank Roma Siugzdaite, Tess Smith, Stepheni Uh, and Jonathan Jones for assistance with data collection, and Mathew H. Davis for materials and assistance in adapting the phonological discrimination task. We thank the two anonymous reviewers for providing constructive feedback to the paper.

Funding The Resilience in Education and Development (RED) Study is supported by grant TWCF0159 from the Templeton World Charity Foundation, and by the UK Medical Research Council.

\section{Compliance with ethical standards}

Declaration of Conflicting Interests The authors declare that there are no conflicts of interest with respect to the authorship or the publication of this article.

Open Practices Materials and analysis scripts are publicly accessible (www.osf.io/xhejz/). Due to ethical constraints, the raw data is only shared on request. A synthetic dataset is provided in the same repository.

Open Access This article is licensed under a Creative Commons Attribution 4.0 International License, which permits use, sharing, adaptation, distribution and reproduction in any medium or format, as long as you give appropriate credit to the original author(s) and the source, provide a link to the Creative Commons licence, and indicate if changes were made. The images or other third party material in this article are included in the article's Creative Commons licence, unless indicated otherwise in a credit line to the material. If material is not included in the article's Creative Commons licence and your intended use is not permitted by statutory regulation or exceeds the permitted use, you will need to obtain permission directly from the copyright holder. To view a copy of this licence, visit http://creativecommons.org/licenses/by/4.0/.

\section{References}

AERA, APA, \& NCME. (2014). The Standards for Educational and Psychological Testing. Washington, DC: American Educational Research Association.

Akshoomoff, N., Newman, E., Thompson, W. K., McCabe, C., Bloss, C. S., Chang, L., ... Jernigan, T. L. (2014). The NIH Toolbox Cognition Battery: Results from a large normative developmental sample (PING). Neuropsychology, 28(1), 1-10. https://doi.org/10. 1037/neu0000001

Alloway, T. P. (2007). Automated working memory assessment. London: Pearson.

GL Assessment. (2012). Cognitive abilities test (4th ed.). London.

Attali, Y., \& Arieli-Attali, M. (2015). Gamification in assessment: Do points affect test performance? Computers \& Education, 83, 5763. https://doi.org/10.1016/j.compedu.2014.12.012

Azah, N., Aziz, A., Syuhada, N., \& Sin, M. (2014). Selection of Touch Gestures for Children's Applications: Repeated Experiment to Increase Reliability. International Journal of Advanced Computer Science and Applications, 5(4), 97-102. https://doi.org/10.14569/ IJACSA.2014.050415

Bates, D., Mächler, M., Bolker, B., \& Walker, S. (2015). Fitting linear mixed-effects models using lme4. Journal of Statistical Software, 67(1). https://doi.org/10.18637/jss.v067.i01

Beckmann, N., Beckmann, J. F., \& Elliott, J. G. (2009). Self-confidence and performance goal orientation interactively predict performance in a reasoning test with accuracy feedback. Learning and Individual 
Differences, 19(2), 277-282. https://doi.org/10.1016/j.lindif.2008. 09.008

Bennett, A. E., Power, T. J., Eiraldi, R. B., Leff, S. S., \& Blum, N. J. (2009). Identifying Learning Problems in Children Evaluated for ADHD: The Academic Performance Questionnaire. Pediatrics, 124(4), e633-e639. https://doi.org/10.1542/peds.2009-0143

Betz, N. E. (1977). Effects of Immediate Knowledge of Results and Adaptive Testing on Ability Test Performance. Applied Psychological Measurement, 1(2), 259-266. https://doi.org/10. 1177/014662167700100212

Bhavnani, S., Mukherjee, D., Dasgupta, J., Verma, D., Parameshwaran, D., Divan, G., Sharma, K.K., Thiagarajan, T. \& Patel, V. (2019). Development, feasibility and acceptability of a gamified cognitive DEvelopmental assessment on an E-Platform (DEEP) in rural Indian pre-schoolers-a pilot study. Global Health Action, 12(1). https://doi. org/10.1080/16549716.2018.1548005

Bignardi, G., Dalmaijer, E., Anwyl-Irvine, A., Smith, T. A., Siugzdaite, R., Uh, S., \& Astle, D. (2020). Increase in childhood depression during the COVID-19 lockdown in the UK.

Brankaer, C., Ghesquière, P., \& De Smedt, B. (2017). Symbolic magnitude processing in elementary school children: A group administered paper-and-pencil measure (SYMP Test). Behavior Research Methods, 49(4), 1361-1373. https://doi.org/10.3758/s13428-0160792-3

Bridges, D., Pitiot, A., MacAskill, M. R., \& Peirce, J. W. (2020). The timing mega-study: comparing a range of experiment generators, both lab-based and online. Peer $J, 8$, e9414. https://doi.org/10. 7717/peerj.9414

Brinkhuis, M., Cordes, W., \& Hofman, A. (2020). Governing games Adaptive game selection in the Math Garden. ITM Web of Conferences, 33, 03003. https://doi.org/10.1051/itmconf/ 20203303003

Button, K. S., Ioannidis, J. P. A., Mokrysz, C., Nosek, B. A., Flint, J., Robinson, E. S. J., \& Munafò, M. R. (2013). Power failure: why small sample size undermines the reliability of neuroscience. Nature Reviews Neuroscience, 14(5), 365-376. https://doi.org/10.1038/ nrn3475

Van Buuren, S.\& Groothuis-Oudshoorn, K. (2011). mice: Multivariate Imputation by Chained Equations in R. Journal of Statistical Software, 45(3). https://doi.org/10.18637/jss.v045.i03

Chalmers, R. P. (2012). mirt: A Multidimensional Item Response Theory Package for the R Environment. Journal of Statistical Software, 48(6). https://doi.org/10.18637/jss.v048.i06

Chapman, C. D., Benedict, C., \& Schiöth, H. B. (2018). Experimenter gender and replicability in science. Science Advances, 4(1), e1701427. https://doi.org/10.1126/sciadv.1701427

Dalmaijer, E.S., Van der Stigchel, S., Nijboer, T.C.W. et al. (2015). CancellationTools: All-in-one software for administration and analysis of cancellation tasks. Behav Res 47, 1065-1075. https://doi.org/ 10.3758/s13428-014-0522-7

Dalmaijer, E., Bignardi, G., Anwyl-Irvine, A., Smith, T. A., Siugzdaite, R., Uh, S., ... \& Astle, D. (2019). Direct and indirect links between children's socio-economic status and education: Pathways via mental health, attitude, and cognition. Chicago.

Dalmaijer, E. S., Nord, C. L., Bignardi, G., Anwyl-Irvine, A. L., Siugzdaite, R., Smith, T. A., Uh, S., Billing, A. \& Astle, D. (2020, February 7). Cognitive profile partly mediates allergy-associated decrease in mental health. MedRxiv. https://doi.org/10.1101/2020. 02.01.20019778

Davis, M. H., Evans, S., McCarthy, K., Evans, L., Giannakopoulou, A., \& Taylor, J. (2019). Lexical learning shapes the development of speech perception until late adolescence. https://doi.org/10.31234/ Osf.Io/Ktsey.10.31234/osf.io/ktsey

Davison, A. C., \& Hinkley, D. V. (2019). boot: Bootstrap R (S-Plus) Functions. R package version 1.3-23.
Delgado, A. R., \& Prieto, G. (2003). The effect of item feedback on multiple-choice test responses. British Journal of Psychology, 94(1), 73-85. https://doi.org/10.1348/000712603762842110

DeWind, N. K., \& Brannon, E. M. (2016). Significant Inter-Test Reliability across Approximate Number System Assessments. Frontiers in Psychology, 7(310). https://doi.org/10.3389/fpsyg. 2016.00310

Dillon, M. R. (2020, September 12). Online labs: under development. BOLD. https://bold.expert/online-labs-under-development/

Dockterman, D., Petscher, Y., McAfee, A., Klopfer, E., Osterweil, S., \& Diefentheler, C. (2020, May 21). Gaming Considerations for Educational Assessments. PsyArXiv. https://doi.org/10.31234/osf. io/en23t

Enkavi, A. Z., Eisenberg, I. W., Bissett, P. G., Mazza, G. L., MacKinnon, D. P., Marsch, L. A., \& Poldrack, R. A. (2019). Large-scale analysis of test-retest reliabilities of self-regulation measures. Proceedings of the National Academy of Sciences, 116(12), 5472-5477. https://doi. org/10.1073/pnas.1818430116

Farrell, S., \& Lewandowsky, S. (2018). Computational modeling of cognition and behavior. Cambridge: Cambridge University Press.

Flake, J. K., \& Fried, E. I. (2019, January 17). Measurement Schmeasurement: Questionable Measurement Practices and How to Avoid Them. PsyArXiv. https://doi.org/10.31234/osf.io/hs7wm

Gaab, N. (2019). How can we ensure that every child will learn to read? The need for a global, neurodevelopmental perspective. https:// dyslexiaida.org/how-can-we-ensure-that-every-child-will-learntoread-the-need-for-a-globalneurodevelopmental-perspective/

Gebuis, T., \& Reynvoet, B. (2011). Generating nonsymbolic number stimuli. Behavior Research Methods, 43(4), 981-986. https://doi. org/10.3758/s13428-011-0097-5

Gregory, R. J. (2014). Psychological Testing: History, Principles, and Applications (7th ed.). Harlow: Pearson Education.

Grice, J. W. (2001). Computing and evaluating factor scores. Psychological Methods, 6(4), 430-450. https://doi.org/10.1037/ 1082-989X.6.4.430

Haines, N., Kvam, P., Irving, L., Beauchaine, T. P., Pitt, M. A., Ahn, W., \& Turner, B. (2020). Learning from the Reliability Paradox: How Theoretically Informed Generative Models Can Advance the Social, Behavioral, and Brain Sciences. https://doi.org/10.31234/osf.io/ $\mathrm{xr} 7 \mathrm{y} 3$

Harrell, T. H., Honaker, L. M., Hetu, M., \& Oberwager, J. (1987). Computerized versus traditional administration of the multidimensional aptitude battery-verbal scale: An examination of reliability and validity. Computers in Human Behavior, 3(2), 129-137. https://doi.org/10.1016/0747-5632(87)90017-3

Harrison, P. M. C., Collins, T., \& Müllensiefen, D. (2017). Applying modern psychometric techniques to melodic discrimination testing: Item response theory, computerised adaptive testing, and automatic item generation. Scientific Reports, 7(1), 3618. https://doi.org/10. 1038/s41598-017-03586-z

Hedge, C., Powell, G., \& Sumner, P. (2018). The reliability paradox: Why robust cognitive tasks do not produce reliable individual differences. Behavior Research Methods, 50(3), 1166-1186. https:// doi.org/10.3758/s13428-017-0935-1

Hofman, A. D., Kievit, R., Stevenson, C., Molenaar, D., Visser, I., \& van der Maas, H. (2018). The dynamics of the development of mathematics skills: A comparison of theories of developing intelligence. OSF Preprints. https://doi.org/10.31219/osf.io/xa2ft

Holmes, J., Bryant, A., \& Gathercole, S. E. (2019). Protocol for a transdiagnostic study of children with problems of attention, learning and memory (CALM). BMC Pediatrics, 19(1), 10. https://doi. org/10.1186/s12887-018-1385-3

Howard, S. J., \& Melhuish, E. (2017). An Early Years Toolbox for Assessing Early Executive Function, Language, Self-Regulation, and Social Development: Validity, Reliability, and Preliminary 
Norms. Journal of Psychoeducational Assessment, 35(3), 255-275. https://doi.org/10.1177/0734282916633009

Jackson, D. N. (1998). Multidimensional aptitude battery II: manual. Sigma Assessment Systems Port Huron, MI.

Jacobsen, T. N., Nohr, E. A., \& Frydenberg, M. (2010). Selection by socioeconomic factors into the Danish National Birth Cohort. European Journal of Epidemiology, 25(5), 349-355. https://doi. org/10.1007/s10654-010-9448-2

Johnson, W., Bouchard, T. J., Krueger, R. F., McGue, M., \& Gottesman, I. I. (2004). Just one g: consistent results from three test batteries. Intelligence, 32(1), 95-107. https://doi.org/10.1016/S01602896(03)00062-X

Jorgensen, T. D., Pornprasertmanit, S., Schoemann, A. M., \& Rosseel, Y. (2018). semTools: Useful tools for structural equation modeling. R Package Version 0.5-1

Joseph, T. N., Hughes, R. W., Sörqvist, P., \& Marsh, J. E. (2018). Differences in Auditory Distraction between Adults and Children: A Duplex-mechanism Approach. Journal of Cognition, 1(1). https:// doi.org/10.5334/joc. 15

Kanerva, K., Kiistala, I., Kalakoski, V., Hirvonen, R., Ahonen, T., \& Kiuru, N. (2019). The feasibility of working memory tablet tasks in predicting scholastic skills in classroom settings. Applied Cognitive Psychology, 33: 1224-1237. https://doi.org/10.1002/acp. 3569

Karr, J. E., Areshenkoff, C. N., Rast, P., Hofer, S. M., Iverson, G. L., \& Garcia-Barrera, M. A. (2018). The unity and diversity of executive functions: A systematic review and re-analysis of latent variable studies. Psychological bulletin, 144(11), 1147-1185. https://doi. org/10.1037/bul0000160

Keiding, N., \& Louis, T. A. (2016). Perils and potentials of self-selected entry to epidemiological studies and surveys. Journal of the Royal Statistical Society: Series A (Statistics in Society), 179(2), 319-376. https://doi.org/10.1111/rssa.12136

Kuperman, V., Stadthagen-Gonzalez, H., \& Brysbaert, M. (2013). Erratum to: Age-of-acquisition ratings for 30,000 English words. Behavior Research Methods, 45(3), 900-900. https://doi.org/10. 3758/s13428-013-0348-8

Ling, G., Attali, Y., Finn, B., \& Stone, E. A. (2017). Is a Computerized Adaptive Test More Motivating Than a Fixed-Item Test? Applied Psychological Measurement, 41(7), 495-511. https://doi.org/10. $1177 / 0146621617707556$

Lissitz, R. (2009). Introduction. In R. Lissitz (Ed.), The Concept of Validity: Revisions, New Directions, and Applications (pp. 1-18). Charlotte, NC: Information Age Publishing Inc.

Luciano, M., Wright, M. J., Geffen, G. M., Geffen, L. B., Smith, G. A., Evans, D. M., \& Martin, N. G. (2003). A genetic two-factor model of the covariation among a subset of Multidimensional Aptitude Battery and Wechsler Adult Intelligence Scale-Revised subtests. Intelligence, 31(6), 589-605. https://doi.org/10.1016/S01602896(03)00057-6

Lumsden, J., Edwards, E. A., Lawrence, N. S., Coyle, D., \& Munafò, M. R. (2016). Gamification of Cognitive Assessment and Cognitive Training: A Systematic Review of Applications and Efficacy. JMIR Serious Games, 4(2), e11. https://doi.org/10.2196/games. 5888

Markus, K. A., \& Borsboom, D. (2013). Frontiers of test validity theory: Measurement, causation, and meaning. New York: Routledge.

Mayer, R. E. (2011). Intelligence and Achievement. In R. J. Sternberg \& S. B. Kaufman (Eds.), The Cambridge Handbook of Intelligence (pp. 738-747). Cambridge: Cambridge University Press. https:// doi.org/10.1017/CBO9780511977244.037

McCredie, M. N., \& Morey, L. C. (2019). Who Are the Turkers? A Characterization of MTurk Workers Using the Personality Assessment Inventory. Assessment, 26(5), 759-766. https://doi. org/10.1177/1073191118760709
McCrimmon, A. W., \& Smith, A. D. (2013). Review of the Wechsler Abbreviated Scale of Intelligence, Second Edition (WASI-II). Journal of Psychoeducational Assessment, 31(3), 337-341. https:// doi.org/10.1177/0734282912467756

McNeish, D. (2018). Thanks coefficient alpha, we'll take it from here. Psychological Methods, 23(3), 412-433. https://doi.org/10.1037/ met0000144

Millsap, R. E., \& Kim, H. (2018). Factorial invariance across multiple populations in discrete and continuous data. in the wiley handbook of psychometric testing (pp. 847-884). Chichester, UK: John Wiley \& Sons, Ltd. https://doi.org/10.1002/9781118489772.ch26

Ministry of Housing Communities and Local Government. (2019). The English Indices of Deprivation 2019 (IoD2019). London.

Munafò, M. R., Nosek, B. A., Bishop, D. V. M., Button, K. S., Chambers, C. D., Percie du Sert, N., ... Ioannidis, J. P. A. (2017). A manifesto for reproducible science. Nature Human Behaviour, 1(1), 0021. https://doi.org/10.1038/s41562-016-0021

Murphy, K. R., \& Davidshofer, C. O. (2004). Psychological Testing. Principles and Applications (Pearson Ed). Harlow.

Nowok, B., Raab, G. M., \& Dibben, C. (2016). synthpop : Bespoke Creation of Synthetic Data in R. Journal of Statistical Software, 74(11). https://doi.org/10.18637/jss.v074.i11

Nüst, D., Eddelbuettel, D., Bennett, D., Cannoodt, R., Clark, D., Daróczi, G., Edmondson, M., Fay, C., Hughes, E., Lopp, S., Marwick, B., Nolis, H., Nolis, J., Ooi, H., Ram, K., Ross, N., Shepherd, L., Turaga, N., Willis, C., Xiao, N., \& Petegem, C.V. (2020, January 29). The rockerverse: packages and applications for containerization with r. https://doi.org/10.32614/RJ-2020-007

Odic, D., \& Starr, A. (2018). An Introduction to the Approximate Number System. Child Development Perspectives, 12(4), 223 229. https://doi.org/10.1111/cdep.12288

Ohtani, K. (2000). Bootstrapping R2 and adjusted R2 in regression analysis. Economic Modelling, 17(4), 473-483. https://doi.org/10.1016/ S0264-9993(99)00034-6

Open Science Collaboration. (2015). Estimating the reproducibility of psychological science. Science, 349(6251), aac4716. https://doi. org/10.1126/science.aac4716

Piatt, C., Coret, M., Choi, M., Volden, J., \& Bisanz, J. (2016). Comparing Children's Performance on and Preference for a Number-Line Estimation Task: Tablet Versus Paper and Pencil. Journal of Psychoeducational Assessment, 34(3), 244-255. https://doi.org/10. 1177/0734282915594746

Pitchford, N. J., \& Outhwaite, L. A. (2016). Can touch screen tablets be used to assess cognitive and motor skills in early years primary school children? A cross-cultural study. Frontiers in Psychology, 7(OCT), 1-14. https://doi.org/10.3389/fpsyg.2016.01666

Pronk, T., Wiers, R. W., Molenkamp, B., \& Murre, J. (2020). Mental chronometry in the pocket? Timing accuracy of web applications on touchscreen and keyboard devices. Behavior Research Methods, 52(3), 1371-1382. https://doi.org/10.3758/s13428-019-01321-2

Putnick, D. L., \& Bornstein, M. H. (2016). Measurement invariance conventions and reporting: The state of the art and future directions for psychological research. Developmental Review, 41, 71-90. https://doi.org/10.1016/j.dr.2016.06.004

Revelle, W. (2018). psych: Procedures for Psychological, Psychometric, and Personality Research. Evanston, Illinois. Retrieved from https:// cran.r-project.org/package $=$ psych

Revelle, W., \& Condon, D. M. (2018). Reliability. In The Wiley Handbook of Psychometric Testing (pp. 709-749). Chichester, UK: John Wiley \& Sons, Ltd. https://doi.org/10.1002/ 9781118489772.ch23

Rey-Mermet, A., Gade, M., Souza, A. S., von Bastian, C. C., \& Oberauer, K. (2019). Is executive control related to working memory capacity and fluid intelligence?. Journal of experimental psychology. General, 148(8), 1335-1372. https://doi.org/10.1037/xge0000593 
Ritchie, S. J., \& Tucker-Drob, E. M. (2018). How much does education improve intelligence? A meta-analysis. Psychological Science, 29(8), 1358-1369.

Robinson, S. J., \& Brewer, G. (2016). Performance on the traditional and the touch screen, tablet versions of the Corsi Block and the Tower of Hanoi tasks. Computers in Human Behavior, 60, 29-34. https://doi. org/10.1016/j.chb.2016.02.047

Röer, J. P., Bell, R., Körner, U., \& Buchner, A. (2018). Equivalent auditory distraction in children and adults. Journal of Experimental Child Psychology, 172, 41-58. https://doi.org/10.1016/j.jecp.2018. 02.005

Rosseel, Y. (2012). lavaan: An R Package for Structural Equation Modeling. Journal of Statistical Software, 48(2). https://doi.org/10. 18637/jss.v048.i02

Roth, B., Becker, N., Romeyke, S., Schäfer, S., Domnick, F., \& Spinath, F. M. (2015). Intelligence and school grades: A meta-analysis. Intelligence, 53, 118-137. https://doi.org/10.1016/j.intell.2015.09. 002

Rouder, J., Kumar, A., \& Haaf, J. M. (2019). Why most studies of individual differences with inhibition tasks are bound to fail.

Sakshaug, J. W., Schmucker, A., Kreuter, F., Couper, M. P., \& Singer, E. (2016). Evaluating Active (Opt-In) and Passive (Opt-Out) Consent Bias in the Transfer of Federal Contact Data to a Third-Party Survey Agency. Journal of Survey Statistics and Methodology, 4(3), 382416. https://doi.org/10.1093/jssam/smw020

Semmelmann, K., Nordt, M., Sommer, K., Röhnke, R., Mount, L., Prüfer, H., Terwiel, S., Meissner, T. W., Koldewyn, K., \& Weigelt, S. (2016). U Can Touch This: How Tablets Can Be Used to Study Cognitive Development. Frontiers in psychology, 7, 1021. https://doi.org/10.3389/fpsyg.2016.01021

Silverman, I. W., \& Rose, A. P. (1982). Compensation and conservation. Psychological Bulletin, 91(1), 80-101. https://doi.org/10.1037/ 0033-2909.91.1.80

Smets, K., Sasanguie, D., Szücs, D., \& Reynvoet, B. (2015). The effect of different methods to construct non-symbolic stimuli in numerosity estimation and comparison. Journal of Cognitive Psychology, 27(3), 310-325. https://doi.org/10.1080/20445911.2014.996568

Stanovich, K. E., Cunningham, A. E., \& Cramer, B. B. (1984). Assessing phonological awareness in kindergarten children: Issues of task comparability. Journal of experimental child psychology, 38(2), 175-190.

Styck, K. M., \& Walsh, S. M. (2016). Evaluating the prevalence and impact of examiner errors on the Wechsler scales of intelligence:
A meta-analysis. Psychological Assessment, 28(1), 3-17. https://doi. org/10.1037/pas0000157

Thompson, W. K., Barch, D. M., Bjork, J. M., Gonzalez, R., Nagel, B. J., Nixon, S. J., \& Luciana, M. (2019). The structure of cognition in 9 and 10 year-old children and associations with problem behaviors: Findings from the ABCD study's baseline neurocognitive battery. Developmental Cognitive Neuroscience, 36, 100606. https://doi.org/ 10.1016/j.dcn.2018.12.004

Thorndike, R. L. (1987). Stability of factor loadings. Personality and Individual Differences, 8(4), 585-586. https://doi.org/10.1016/ 0191-8869(87)90224-8

Ushey, K. (2020). renv: Project Environments. R package version 0.9.3. https:/CRAN.R-project.org/package $=$ renv

Vernon, P. A. (1989). The generality of g. Personality and Individual Differences, 10(7), 803-804. https://doi.org/10.1016/01918869(89)90129-3

Warne, R. T., \& Burningham, C. (2019). Spearman's g found in 31 nonWestern nations: Strong evidence that $\mathrm{g}$ is a universal phenomenon. Psychological Bulletin, 145(3), 237-272. https://doi.org/10.1037/ bul0000184

Wassenaar, T. M., Wheatley, C. M., Beale, N., Salvan, P., Meaney, A., Possee, J. B., ... Johansen-Berg, H. (2019). Effects of a programme of vigorous physical activity during secondary school physical education on academic performance, fitness, cognition, mental health and the brain of adolescents (Fit to Study): study protocol for a cluster-randomised trial. Trials, 20(1), 189. https://doi.org/10. 1186/s13063-019-3279-6

Wasserman, J. D. (2018). A history of intelligence assessment: The unfinished tapestry. In Contemporary intellectual assessment: Theories, tests, and issues, 4th ed. (pp. 3-55). New York, NY, US:The Guilford Press.

Weintraub, S., Dikmen, S. S., Heaton, R. K., Tulsky, D. S., Zelazo, P. D., Bauer, P. J., ... Gershon, R. C. (2013). Cognition assessment using the NIH Toolbox. Neurology, 80(Issue 11, Supplement 3), S54S64. https://doi.org/10.1212/WNL.0b013e3182872ded

Winding, T. N., Andersen, J. H., Labriola, M., \& Nohr, E. A. (2014). Initial non-participation and loss to follow-up in a Danish youth cohort: Implications for relative risk estimates. Journal of Epidemiology and Community Health, 68(2), 137-144. https://doi. org/10.1136/jech-2013-202707

Publisher's note Springer Nature remains neutral with regard to jurisdictional claims in published maps and institutional affiliations. 